$9 \quad *$ the authors contributed equally to the study.

10

13 Email: david.berger@ebc.uu.se

14

\# Corresponding author

\title{
Sexual selection and the evolution of male and female cognition: a test using experimental evolution in seed beetles
}

\author{
Julian $\operatorname{Baur}^{1 *}$, Jean d'Amour $^{1 *} \&$ David Berger ${ }^{1 \#}$
}

1. Department of Ecology and Genetics, Animal Ecology program. Uppsala University.

Keywords: Sexual selection, cognition, host choice, mate choice, mating mind, good genes, sexual antagonism, intralocus sexual conflict. 


\section{Abstract}

19 "The mating mind hypothesis", originally aimed at explaining human cognition, holds that

20 the socio-sexual environment shapes cognitive abilities among animals. Similarly, general

21 sexual selection theory predicts that mate competition should benefit individuals carrying

22 "good genes" with beneficial pleiotropic effects on general cognitive ability. However, few

23 experimental studies have evaluated these related hypotheses due to difficulties of performing

24 direct tests in most taxa. Here we harnessed the empirical potential of the seed beetle study

25 system to investigate the role of sexual selection and mating system in the evolution of

26 cognition. We evolved replicate lines of beetle under enforced monogamy (eliminating sexual

27 selection) or polygamy for 35 generations and then challenged them to locate and

28 discriminate among mating partners (male assays) or host seeds (female assays). To assess

29 learning, the same beetles performed the task in three consecutive rounds. All lines learned

30 the task, improving both within and between trails. Moreover, polygamous males

31 outperformed monogamous males. However, there were no differences in the rate of learning

32 between males of the two regimes, and polygamous females showed no improvement in host

33 search, and even signs of reduced learning. Hence, while sexual selection was a potent factor

34 that increased cognitive performance in mate search, it did not lead to the general increase in

35 cognitive abilities expected under the "mating mind" hypothesis or general "good genes"

36 theory. Our results highlight sexually antagonistic (balancing) selection as a potential force

37 maintaining genetic variation in cognitive traits. 


\section{$38 \quad$ Background}

39 Cognitive traits allow for behavioural plasticity which can fundamentally change

40 evolutionary dynamics and the mode of, and limits to, adaptation ${ }^{1-4}$. Cognitive abilities also

41 vary widely among animal taxa and there are many hypotheses aimed at explaining this

42 interspecific variation. Most evolutionary explanations typically emphasize the importance of

43 trade-offs and species ecology in shaping cognition ${ }^{5-10}$. One such hypothesis is the idea that

44 the social system of a species is particularly important in shaping cognitive abilities ${ }^{11}$. Indeed,

45 the complex social structure of human societies has been suggested as a main driver of our

46 species' intelligence ${ }^{12}$. This idea has also been expanded and popularized to include the

47 socio-sexual environment, advocating the view that sexual selection and competition over

48 mating partners has been an important factor contributing to human cognition, which only

49 later allowed the successful colonization of new environments and unprecedented cultural

50 innovations of our species ("The mating mind hypothesis") ${ }^{13,14}$.

52 The arena for socio-sexual interactions and associated cognitive decision making appear

53 somewhat different in humans compared to other animals. However, it is perhaps only from

54 an anthropocentric perspective that these differences can be seen as larger than those between

55 any other two animals with different mating systems ${ }^{15}$, suggesting that the mating mind

56 hypothesis could be generalized to explain variation among non-human taxa ${ }^{16}$. Indeed, the

57 idea that sexual selection requires cognitive abilities has been widely explored (e.g. ${ }^{5,14,16-18}$ ).

58 However, comparative evidence for a direct link between mating system variation and animal

59 cognition is mixed ${ }^{16,17,19}$. For example, in primates monogamy rather than polygamy is

60 associated with larger brain $\operatorname{size}^{20}$, providing evidence against the hypothesis that sexual

61 selection leads to increased cognition but not excluding that complex social structure is

62 important, given that maintaining monogamous pair-bonds may be cognitively demanding ${ }^{5,11}$. 
63 In both primates ${ }^{6}$ and birds ${ }^{21}$, environmental complexity is more strongly associated with

64 brain size than the social system of the species. Moreover, in bats, males of species where

65 females are promiscuous tend to have smaller brains but larger testes compared to species

66 where females exhibit mate fidelity ${ }^{22}$, suggesting that sexual selection may even lead to

67 decreased cognitive ability via trade-offs with expensive secondary sexual traits.

69 These comparative studies provide a less than convincing case for an important role of the 70 mating system in the evolution of cognition. Given the many, potentially confounding, 71 factors associated with animal sociality, mating systems and ecology, it may be that 72 controlled experiments are needed to compliment comparative methods ${ }^{23}$ and evaluate the 73 generality of the mating mind hypothesis as applied to animals in general. The question thus 74 remains whether sexual selection and mating system variation generally are important for the 75 evolution of cognition in its widest definition - i.e. does selection for cognitive performance 76 during mating competition lead to greater cognition when applied to other tasks or behaviors?

78 The mating mind hypothesis is routed in general sexual selection theory ${ }^{17,24}$, which holds that 79 competition over access to mating partners should select for males that carry genomes free of 80 deleterious mutations (i.e. males carrying the "good genes") ${ }^{25}$. Since most new mutations are 81 thought to be deleterious and have wide ranging pleiotropic effects on fitness related traits ${ }^{26}$, 82 it follows that males that are successful in mating competition should on average be superior 83 performers, and pass on these "good genes" to both sons and daughters ${ }^{27}$. Hence, the mating 84 mind and good genes hypothesis make largely parallel predictions of an association between 85 the mating system and general cognitive ability. 
87 Here we tested the role of sexual selection and mating system variation in the evolution of cognition by harnessing the empirical potential of experimental evolution and the seed beetle study system. We evolved replicate evolution lines of Callosobruchus maculatus beetles

90 under enforced monogamy (excluding sexual selection) or natural polygamy (including high

91 levels of sexual competition and mate choice) for 35 generations. We then subjected these

92 lines to a cognitively challenging spatial and chemo-sensory task, composed of mate finding 93 and discrimination in males, and host seed finding and discrimination in females. We also 94 assessed cognitive learning for both tasks (in males and females respectively) by letting the 95 same beetles perform the task in three consecutive rounds with interspaced acclimation 96 periods. Hence, our design allowed us to assess whether experimental evolution had led to 97 improved cognitive performance in the specific task (male mate search and discrimination) 98 known to be under differential selection in the monogamy and polygamy regime, and then to 99 explore whether evolution under the contrasting mating systems had led to genetic changes in general cognitive ability inferred from i) improved female host search and discrimination, and ii) improved cognitive learning in the tasks, assessed in respective sex.

\section{Methods}

\section{Study species}

C. maculatus seed beetles are common pests of legumes (Fabaceae) in Africa and Asia. Females lay eggs on seeds and larvae burrow into the seed where the entire development occurs $^{28}$. Beetles emerging from seeds are reproductively mature and require neither water nor food to reproduce (e.g. ${ }^{28,29}$ ). Adults typically die in 7-14 days after emergence in the absence of food or water (e.g. $\left.{ }^{30}\right)$. 
111 Sexual selection is intense in this species, including both pre- and post-copulatory 112 processes $^{31-36}$. Sexual selection is thus likely to put demands on both male and female 113 cognitive abilities associated with mate choice, including assessing sex, age, body size, 114 phenotypic quality, as well as mating status of potential mating partners, as all these choices 115 can potentially influence reproductive success ${ }^{28,31,37-39}$. In the lab environment males search 116 for females among beans, putting additional demands on male spatial orientation and use of 117 olfactory cues to locate and discriminate females ${ }^{40}$, who when mated often hide amongst the 118 beans to escape costly re-mating attempts by males ${ }^{34-36}$.

Female host plant search and discrimination is complex. C. maculatus has a wide repertoire of fabaceus host plants $^{41}$ but females have a clear host hierarchy and preference, and they 122 discriminate between high quality and low quality species (e.g. ${ }^{42,43}$ ). In the lab environment 123 females are typically presented with beans from one host species, but need to discriminate among good and bad quality seeds as well as against egg-laden seeds, as high larval density or poor quality host seeds limit both survival and size at maturity of offspring, and thus come at substantial fitness $\operatorname{costs}^{38,43}$.

The experimental evolution lines (see below) come from a genetic stock recently isolated from the wild ${ }^{44}$. C. maculatus utilizes both natural habitats, where host plant patches used for 130 both egg laying and adult nectar feeding are more widely distributed, as well as grain storages, where adult food is absent but egg laying substrate and population density is 132 higher ${ }^{38,41,43}$. Thus, selection on spatial cognition and chemosensory cues is likely always strong in natural populations, but may take different forms, which is predicted to maintain genetic variation in the cognitive traits under study, as seen for other characters related to these alternative environments (e.g. ${ }^{45}$ ). 


\section{Polygamous and Monogamous experimental evolution lines}

138 The lines used in this study are thoroughly described in Martinossi-Allibert et al. ${ }^{46}$. In brief,

139 the lines and the outbred base population from which they originate were maintained under

140 controlled temperature $\left(29^{\circ} \mathrm{C}\right)$, humidity $(50 \% \mathrm{RH})$ and light cycle $(12 \mathrm{~L}: 12 \mathrm{D})$, and reared on

141 the preferred host plant ${ }^{41}$ Vigna unguiculata (black-eyed bean). We used six lines in this

142 study; three replicate lines evolving under enforced monogamy (removing sexual selection),

143 and three lines evolving under polygamy in the natural lab environment which applies sexual

144 selection by allowing pre- and post-copulatory mate competition and choice, in addition to

145 the fecundity and viability selection acting in the monogamy regime. There are two more

146 lines in the study by Martinossi-Allibert et al. from a third evolution regime which applied

147 sexual selection on males while excluding selection on adult females all together. However,

148 since our hypotheses were most straightforward to test by comparing the effect of adding

149 sexual selection (polygamy regime) to an already natural socio-sexual mating system

150 (monogamy regime), we did not include these two lines in this study.

152 Effective population size in each regime was kept approximately equal $\left(\mathrm{N}_{\mathrm{e}}=150, \mathrm{~N}_{\text {monogamy }}=\right.$ $153246, \mathrm{~N}_{\text {polygamy }}=300$ ) and the number of beans provided as egg laying substrate in each 154 regime was standardized to give the same, relatively low, juvenile density (2-4 eggs/bean) to 155 minimize (and equalize) larval competition ${ }^{46}$. The polygamy and monogamy regime show 156 differences in line with good genes effects of sexual selection: already following 16-20 157 generations of experimental evolution, polygamy lines showed higher lifetime reproductive 158 success as well as population fitness compared to monogamy lines ${ }^{46}$. 
160

161

162

163

\section{Cognitive performance of males in the mate searching task}

We measured focal males' (i.e. derived from one of the evolution lines) ability to localise and discriminate females in a spatially complex arena made up by a petri dish measuring $150 \mathrm{~mm}$ in diameter containing reference beetles of both sexes (Fig. 1). The reference beetles originated from the original base population from which the selection lines were derived. Virgin reference beetles, $0-24 \mathrm{~h}$ old, were frozen at $-20^{\circ} \mathrm{C}$ and defrosted just previous to a trail and glued to the arena (with their ventral side facing downwards), making sure that the focal beetles did not shift their position during the three consecutive behavioural trails. The same reference beetles were used for the full run of three trails before being replaced by new beetles.

The arena floor was covered with paper (Fig. S1) designed to aid spatial memory and learning. The paper had pale red background and a white large circle connected to a white channel leading away from it towards the inner wall of the opposite side of the arena, ending in a smaller circle at which the four focal males were placed at the initiation of each trial (see below). Each large white circle contained four equidistant points where the freshly defrosted reference females were glued. The 'channel' was connected to the circle to easier allow focal males to learn and find the location of females. The remaining pale red background comprised ten equidistant points where the freshly defrosted reference males were glued (Fig. 1). A second type of arena with inversed color scheme was used to control for effects of potential color preferences in the beetles (Fig. S1).

The assays were run on a heating plate set at $30^{\circ} \mathrm{C}$, with six arenas on the plate scored simultaneously. In each arena, 4 focal males were introduced simultaneously. This was for two reasons, i) to increase activity, because beetles become more active in group, and ii) to 
efficiently score as many beetles as possible. Assays were initiated by consecutively introducing the four beetles into the small circle in each of the 6 replicate arenas. This took ca. 60 seconds after which behavioral observations were taken in the same sequential order each minute for the subsequent 10 minutes. Each census time of an arena lasted for 10 seconds before the next arena in line was observed. During the 10 seconds we recorded whether each of the four beetles in the arena were in contact with a reference individual and whether this was a male or a female. This contact usually meant that males were trying to, or even "successfully" mated with both (dead) males and females (males try to mate readily with other males in this species and population ${ }^{40}$ ). The four beetles were thus scored as a group and could at each census time get a score between 0 and 4 for mating attempts on reference males and females.

Our measurements are likely to capture two independent aspects of seed beetle cognition; i) the ability to locate and remember the location of reference individuals in a spatially complex two-dimensional landscape, and ii) via chemo-sensory cues discriminate the sex of the located reference individual (which seems cognitively demanding in seed beetles: ${ }^{40}$, as in many other insects $\left.{ }^{47}\right)$.

\section{Cognitive performance of females in the host searching task}

We measured focal females' ability to find and discriminate among a high quality ( $V$. unguiculata, black-eyed bean) and a low quality (Cicer arietinum: Chick pea) host species ${ }^{41}$ in the exact same type of arena and set-up (Fig. 1, Fig. S1). To make sure that females were motivated to search for hosts, they were mated with conspecific males 24 hours prior to the trials, and prevented from laying eggs by depriving them of host seeds. In contrast to male trails, host seeds did not need to be glued to stay in their place. Host seeds were also removed 
210 between each female trail as eggs were laid readily by females and the presence of eggs on

211 the hosts can affect female egg laying behaviour (heavily laden seeds are more often

212 rejected). We registered female host inspection as behaviour. This inspection behavior was

213 usually in form of females being on top of seeds making tactile contact with or ovipositing on

214 the seed. Females readily laid eggs on both types of hosts during the assays, although this was

215 never quantified. Similarly to the male assays, our scoring of behaviour captures variation in

216 female cognition in terms of i) spatial orientation and memory as well as ii) chemo-sensory

217 cues associated with host discrimination.

\section{Scoring behavior}

220 The observer (JdA) was always blinded to which line and evolution regime that was assayed.

221 Each group of four beetles were scored for their behaviour as a group through three 10 222 minute trails with a 20 minute acclimation period in a $30 \mathrm{~mm}$ diameter petri dish before and 223 in-between each trail. We could thus study overall differences in beetle cognition in terms of 224 the average performance over time in each line and sex, as well as the potential for cognitive 225 learning by looking at the improvement in performance within and between trails. We used two heating plates, each with one of the two color schemes (Fig. S1). The heating plates were run interchangeably (during the other plate's acclimation periods), and each line was scored once on each plate/color scheme per sex, resulting in 12 arena replicates per sex and line.

229 Thus, 48 males and 48 females from each of the 6 lines were scored for behaviour over three consecutive 10 minute trails for a total of 17.280 behavioral observations.

\section{Statistics}

233 Male and female data were of the same form and were first analysed separately in equivalent 234 models. We modelled the response as an "error rate" (the fraction of incorrect choices) over 
235 the trails using a binomially distributed response variable with the levels "male/female" (male 236 assays) or "good host/bad host" (female assays). Time of the trail was included as a covariate 237 that was linearized by taking its natural logarithm prior to analysis. Evolution regime and trail 238 number were analysed as discrete factors. We included interactions among all three of these 239 explanatory variables, where two or three-way interactions including evolution regime would 240 signify differences in performance over time among the two evolution regimes, indicative of 241 an effect of sexual selection on cognitive learning. A main effect of evolution regime, on the 242 other hand, would indicate an effect of sexual selection on general cognitive performance in 243 the given task. Line identity was included as a random effect crossed with the three 244 explanatory variables to account for the true replication of the experiment (being the six line 245 replicates, and not individual observations). We included assay identity (the four beetles run 246 together over consecutive trails) as an additional random effect. We also modelled main 247 effects of heating plate to control for spatial effects in the lab and beetle color preferences. 248 However, this effect was never significant and was ultimately removed from all models.

In addition to the male- and female-specific model, we also looked more formally for sexdifferences in the response of cognitive performance to experimental evolution under alternative mating systems. This was done by recoding the levels of the response variable to "correct" and incorrect" and then running a statistical model with the main effect of "sex" crossed by the three other explanatory variables of interest (time, trail number and evolution regime) and the random effect of line identity.

257 Analyses were carried out with the package $\operatorname{lme} 4^{48}$ in the statistical software R. We used the "bobyqa" optimizer to increase the number of iterations (to 100.000) to achieve convergence 259 of all models. We report type-II P-values based on likelihood ratio tests and $\boldsymbol{\chi}^{2}$-statistics. 
260 Results

\section{Cognitive performance of males}

262 Throughout the time of the trials, males increased the fraction of mounting attempts on 263 females $\left(\chi_{1}^{2}=80.1, \mathrm{P}<0.001\right)$, suggesting that they learned the spatial location of females 264 and then preferred to stay there to try to mate with them. While there was no main effect of 265 trial, there was a strong interaction between trail and time $\left(\chi_{2}^{2}=18.7, \mathrm{P}<0.001\right)$ because males were more efficient in finding females already at the start of the third trail when trained, compared to the first trail when naïve. These results thus demonstrate clear effects of learning on performance in the mate searching task (Fig. 2A \& C, Fig. S2). Males from the polygamy regime were more efficient overall in discriminating the sex of beetles compared to males from the monogamy regime $\left(\chi_{1}^{2}=8.56, \mathrm{P}=0.003\right.$, Fig. $\left.2 \mathrm{~A} \& \mathrm{C}\right)$. This difference was mainly driven by the number of mounting attempts on reference males, which was much higher in males from monogamous lines (Fig. S2). However, there were no significant differences between selection regimes in how mate discrimination changed within or between trails (Fig. 2A \& C, Table S2, Fig S2), indicating that learning was similar in the two evolution regimes (full statistics in Supplementary Table S2).

\section{Cognitive performance of females}

278 Throughout the time of the trials, females decreased the fraction of visits to suboptimal host 279 seeds $\left(\chi^{2}=246.7, \mathrm{P}<0.001\right)$, suggesting that they learned the spatial location of the optimal host and then preferred to stay there to oviposit (Fig. 2B, D, Fig. S3). There was also a strong main effect of trial $\left(\chi_{2}^{2}=99.1, \mathrm{P}<0.001\right)$, as well as a strong interaction between trail and time $\left(\chi_{2}^{2}=33.8, \mathrm{P}<0.001\right)$, signifying that females improved in the host searching task through spatial and/or chemo-sensory learning (Fig. 2B, D, Fig. S3). However, contrary to the superior performance of polygamous lines in the male task, females from the two regimes did not show any overall differences in host search and discrimination (Table S3). This sex- 
difference in the evolutionary response of cognitive performance was statistically significant $\left(\chi_{1}^{2}=5.91, \mathrm{P}=0.015\right.$, Table $\left.\mathrm{S} 4\right)$. There were also no significant two-way interactions between selection regime and time or trail (Table S3), suggesting that female learning was largely similar in the two evolution regimes. There was, however, a marginally nonsignificant three-way interaction between selection regime, trail and time $\left(\chi_{2}^{2}=5.76, \mathrm{P}<\right.$ 0.056, Fig S3). This trend was driven by a pattern where evolution regimes showed very similar performance throughout the first trail as naïve beetles (regime:time interaction: $\chi_{1}^{2}=$ $0.02, \mathrm{P}=0.89$ ), while monogamous lines tended to be better at discriminating between hosts when trained at the start of the third trail, but where this difference between regimes quickly disappeared as the trail went along (regime:time interaction: $\chi_{1}^{2}=4.28, \mathrm{P}=0.039$, Fig. S3). We note that this difference runs counter to the expectation that sexual selection should improve general cognitive abilities (full statistics in Supplementary S3 \& S4).

\section{Sex-specific correlations between cognitive performance and lifetime reproductive success}

We explored the link between cognitive performance and fitness in each sex by calculating correlations between lifetime reproductive success (LRS, reported $\mathrm{in}^{46}$ ) and the measured cognitive traits (error rate in trail 1-3 and a learning score based on the relative reduction in error rate between the first and last trail: [e1-e3] / e1), based on trait means per sex and evolution line replicate. These correlations are graphically depicted in figure 4 . We note that our interpretation here must remain tentative since these correlations are based only on 6 data-points (three replicate lines per mating regime), and hence, cannot be used for rigorous statistical testing. Error rates are highly correlated between the three trails within each sex (r $=0.58-0.96)$, suggesting substantial repeatability in behaviour among the six genotypes. However, error rates and the learning score are very weakly correlated between sexes $(\mathrm{r}=-$ 0.08-0.34), implying that different genes govern mate search in males and host search in 
311 females. Moreover, while male LRS was negatively correlated to male error rates $(r=-0.68$ -

$312-0.59)$ and positively correlated to male learning $(\mathrm{r}=0.59)$ as predicted, it was positively

313 correlated to the female error rate $(r=0.30-0.62)$ and negatively correlated to female learning

$314(\mathrm{r}=-0.51)$. This may suggest genetic conflict between the sexes at loci encoding the studied

315 cognitive traits. Finally, female learning was negatively correlated to female LRS on both the

316 focal ancestral host (black eyed bean; $r=-0.68$ ) as well as an alternative host ${ }^{46}$ (adzuki bean;

$317 \mathrm{r}=-0.71$ ), suggesting that cognitive learning may trade-off against fecundity in females.

\section{Discussion}

320 It is well known that sexual selection can put demands on cognitive abilities related to sexual 321 signalling and mate choice ${ }^{5,16-18}$ and mate search has also been linked to spatial learning in 322 both vertebrates ${ }^{49}$ and insects ${ }^{50}$. For example, in guppies, females from lines selected for 323 larger brains were better at choosing among high and low quality mating partners ${ }^{51}$ and males

324 from the same lines were better at finding mates in a spatial learning task ${ }^{52}$. Similarly, in fruit

325 flies, cognitive learning improves female mate choice ${ }^{18}$. However, whether the mating system 326 can drive species differences in general $\operatorname{cognition}^{13}$ is much more disputed and direct 327 evidence remains scarce. Here we have shown that mating system variation can lead to the 328 evolution of cognitive performance. Males evolving under polygamy were more efficient in 329 directing their mating effort towards females in spatially complex mixed-sex settings. Given 330 that these males also have higher reproductive success than males evolving under 331 monogamy ${ }^{46}$, this suggests that increased cognitive performance in mate search has fitness 332 benefits in males (see also Fig. 4). However, the evolved increase in mate search ability was 333 not accompanied by improved learning or increases in female cognitive performance, as expected under the mating mind hypothesis. 
336 These negative results are readily interpretable as there was sufficient power in our design to

337 detect significant differences in male performance between evolution regimes, as well as to

338 demonstrate substantial improvement in the cognitive tasks through learning in both males

339 and females (Figs. $2 \& 3$ ). Our main results thus imply that good genes processes resulting in

340 overall improvement of cognitive ability may not materialize when sexual selection acts on

341 standing genetic variation, as it did in our experiment. Interestingly, the decreased ability of

342 monogamous males (relative to polygamous males) to avoid directing mating attempts

343 toward other males was drastic and evolved in only 35 generations of relaxed sexual selection

344 (Fig. S2). This fast decrease implies that selection acted on segregating genetic variation with

345 antagonistic pleiotropic effects on other fitness related traits, because i) decreases in

346 monogamous lines due to the accumulation of de novo mutation over such short time frames

347 seem unlikely, and ii) effects of genetic drift should be negligible since effective population

348 size was relatively large $(\mathrm{Ne} \sim 150)$, all three replicate lines for each regime showed parallel

349 divergence (Fig 2A \& C, Fig. 3) and the effect of drift was controlled for in the applied

350 statistical models. Our results thus also pose the question of what maintains such vast

351 amounts of genetic variation in male cognitive behaviour.

353 Our results are in many ways similar to the study by Hollis and Kawecki (2014) on

354 Drosophila melanogaster fruit flies, which is the only other study we know of that has

355 applied experimental evolution and manipulated the mating system to look at effects on

356 cognitive abilities. In their study, evolution under polygamy contributed to the maintenance

357 of mate acquisition abilities in males, but also lead to superior aversive learning - a cognitive

358 task not directly related to the applied sexual selection. While this suggests that sexual

359 selection improved general cognitive abilities, in line with the mating mind hypothesis,

360 polygamous females showed no such increase, and even tendencies for reduced cognitive 
361 performance relative to females from monogamous lines ${ }^{53}$. This is also in line with our

362 results, showing no differences in performance between monogamous and polygamous $C$.

363 maculatus females overall, and a tendency for monogamous females to learn faster (Fig. S3).

Indeed, as an alternative to good genes effects, sexual selection may lead to sex-limited

responses and increased sexual dimorphism in cognition ${ }^{13,19,49,54,55}$. Such an outcome is

expected when males and females experience different selection pressures and genetic constraints are not insurmountable ${ }^{56}$, so that cognitive traits can evolve independently in each $\operatorname{sex}^{19}$. One mechanistic explanation for the sex-specificity observed in this study could be differences in the chemosensory machinery required to successfully identify and discriminate the sex of mating partners and host species (in males and females respectively). Given that collecting and processing such information should require costly development and maintenance of neuroreceptors ${ }^{4,50}$, cognitive performance in mate search and host search may trade-off against each other, if different receptors are employed for the two tasks and these receptors compete for resources, physical space, or downstream cognitive processing of their transmitted information. This hypothesis is in line with the tendency for sexual selection to have positive effects on male mate search but slightly negative effects on female cognitive learning in both our study and Hollis and Kawecki's (2014) study on fruit flies. Moreover, in this population of beetle, female fecundity has previously been shown to be negatively correlated to the accuracy of male sex discrimination ${ }^{40}$, and in this study, female host search and discrimination tended to be negatively correlated to male reproductive success (Fig. 4).

383 These results imply that selection on cognitive traits may sometimes act with opposing forces 384 in the sexes. If in such cases genetic constraints are preventing each sex from evolving 385 independently from the other ${ }^{56,57}$, this type of sexual antagonism ${ }^{58}$ will generate balancing 
selection that can act to maintain allelic variation at genes underpinning cognitive abilities $^{59,60}$. Mechanistically, sexual antagonism over cognitive traits could, for example, arise if males benefit mostly from increasing allocation to one type of chemoreceptor (e.g. increasing accuracy of sex discrimination) while females benefit from allocation to another type of receptor (e.g. increasing accuracy of host discrimination). Sexual antagonism could also arise if the benefit of a specific cognitive ability is limited mainly to one sex while its energetic cost is paid by both sexes, as seen for other types of traits under sexually antagonistic selection $^{40,61,62}$. In this study, female learning correlated negatively with both male and female reproductive success (Fig. 4), in line with this hypothesis.

Indeed, cognitive traits and learning are generally assumed to come with energetic costs. For example, there are cost of developing and using a large brain in vertebrates ${ }^{7,9,63}$, as well as documented costs of memory and allocation to cognitive traits in insects ${ }^{4,50,64-66}$. Similarly, sexually selected traits are themselves expected to be costly ${ }^{17,24,27,67}$, and while some studies have found a positive genetic correlation between primary and secondary sexual traits and brain size (e.g. ${ }^{68,69}$ ), in line with good genes effect, there are also examples of negative correlations (e.g. ${ }^{22}$ ), suggesting that increased sexual selection may sometimes lead to decreases in cognitive traits via energy allocation trade-offs ${ }^{24,70,71}$. The action of such antagonistic pleiotropy within and between sexes to maintain genetic variation could thus be responsible for the substantial amounts of standing genetic variation in male sex discrimination documented here and previously ${ }^{40}$ in this population of $C$. maculatus. The notion that sexually antagonistic selection has played a key role in this process is also supported by previous studies on the population ${ }^{40,44,72,73}$. 
410 Behavioral plasticity can play an important role in deciding species distributions, persistence

411 and modes of adaptation to changing environments, for example by increasing the efficacy of

412 spatial exploration and resource sampling mediating niche matching ${ }^{1-5}$. Cognitive processes

413 are also key in mate choice dynamics and may therefore play a role in speciation ${ }^{74-79}$. The

414 interplay between sexual selection and the evolution of cognition, with special emphasis on

415 potentially underappreciated effects of sexually antagonistic selection on cognitive traits, may

416 therefore have important consequences for evolutionary dynamics and certainly deserves

417 more attention in other study systems. While the mating mind and good genes hypothesis

418 predict a positive association between cognitive ability and the strength of sexual selection to

419 be built up by purifying selection against recurrent deleterious pleiotropic mutations, our

420 study implies that much of the standing genetic variation in cognitive performance upon

421 which evolutionary responses to novel environments rely, will have been moulded and

422 maintained by forces of balancing selection within and between the sexes. This sets the stage

423 for rapid sex-specific responses to changes in ecological and socio-sexual conditions. 


\section{Competing interests}

427 The authors report no competing interests

\section{Ethics statement}

430 All experiments have been carried out within the regulations held by Swedish governmental 431 laws. Actions were taken to reduce the number of beetles used in the study.

\section{Data accessibility:}

434 Data will be uploaded to the Dryad Data Repository upon potential acceptance

\section{Author's contributions}

437 The study was conceived by JB, JdA and DB, and was carried out by JdA and JB. DB 438 analyzed the data and wrote the manuscript. All authors commented on the first draft.

\section{Acknowledgements}

441 We thank Ivain Martinossi-Allibert and Göran Arnqvist who have helped to create the 442 experimental evolution lines. We also like to thank Johanna Liljestrand-Rönn for practical 443 help in the lab.

\section{$445 \quad$ Funding}

446 The study was funded by grant 2015-05233 from the Swedish Research Council, VR, to DB. 


\section{$448 \quad$ References}

449 1. Baldwin, J. M. A New Factor in Evolution. Am. Nat. 30, 441-451 (1896).

450 2. Smith, J. M. When learning guides evolution. Nature 329, 761-762 (1987).

451 3. Sznajder, B., Sabelis, M. W. \& Egas, M. How Adaptive Learning Affects Evolution:

452 Reviewing Theory on the Baldwin Effect. Evol. Biol. 39, 301-310 (2012).

453 4. Snell-Rood, E. C. An overview of the evolutionary causes and consequences of

$454 \quad$ behavioural plasticity. Anim. Behav. 85, 1004-1011 (2013).

455 5. Dukas, R. Evolutionary Biology of Animal Cognition. Annu. Rev. Ecol. Evol. Syst. 35,

$456 \quad 347-374(2004)$.

457 6. Rosati, A. G. Foraging Cognition: Reviving the Ecological Intelligence Hypothesis.

$458 \quad$ Trends Cogn. Sci. 21, 691-702 (2017).

459 7. Aiello, L. C. \& Wheeler, P. The Expensive-Tissue Hypothesis: The Brain and the

460 Digestive System in Human and Primate Evolution. Curr. Anthropol. 36, 199-221

$461 \quad$ (1995).

462 8. Shettleworth, S. J. Animal cognition and animal behaviour. Anim. Behav. 61, 277-286

$463 \quad(2001)$

464 9. Isler, K. \& van Schaik, C. P. The Expensive Brain: A framework for explaining

465 evolutionary changes in brain size. J. Hum. Evol. 57, 392-400 (2009).

466 10. Johnston, T. D. Selective Costs and Benefits in the Evolution of Learning. in Advances in

467 the Study of Behavior (eds. Rosenblatt, J. S., Hinde, R. A., Beer, C. \& Busnel, M.-C.) 12,

468 65-106 (Academic Press, 1982).

469 11. Dunbar, R. I. M. The social brain hypothesis. Evol. Anthropol. Issues News Rev. 6, 178-

$470 \quad 190(1998)$. 
471 12. Lotem, A., Halpern, J. Y., Edelman, S. \& Kolodny, O. The evolution of cognitive

472 mechanisms in response to cultural innovations. Proc. Natl. Acad. Sci. 114, 7915-7922

473 (2017).

474 13. Miller, G. The Mating Mind: How Sexual Choice Shaped the Evolution of Human Nature.

$475 \quad$ (Anchor, 2001).

476 14. Miller, G. F. \& Todd, P. M. Mate choice turns cognitive. Trends Cogn. Sci. 2, 190-198

$477 \quad$ (1998).

478 15. Mendelson, T. C. et al. Cognitive Phenotypes and the Evolution of Animal Decisions.

$479 \quad$ Trends Ecol. Evol. 31, 850-859 (2016).

16. Boogert, N. J., Fawcett, T. W. \& Lefebvre, L. Mate choice for cognitive traits: a review of the evidence in nonhuman vertebrates. Behav. Ecol. 22, 447-459 (2011).

17. Andersson, M. Sexual Selection. (Princeton University Press, 1994).

18. Dukas, R. Learning affects mate choice in female fruit flies. Behav. Ecol. 16, 800-804 (2005).

19. Geary, D. C. Sexual Selection and Sex Differences in Social Cognition. 31

20. Schillaci, M. A. Sexual Selection and the Evolution of Brain Size in Primates. PLoS ONE

22. Pitnick, S., Jones, K. E. \& Wilkinson, G. S. Mating system and brain size in bats. Proc.

21. Sayol, F. et al. Environmental variation and the evolution of large brains in birds. Nat.

23. MacLean, E. L. et al. How does cognition evolve? Phylogenetic comparative psychology. Anim. Cogn. 15, 223-238 (2012). Darwin's Puzzle. (Oxford University Press, 1999). 
25. Tomkins, J. L., Radwan, J., Kotiaho, J. S. \& Tregenza, T. Genic capture and resolving the lek paradox. Trends Ecol. Evol. 19, 323-328 (2004).

26. Lynch, M. et al. Perspective: Spontaneous Deleterious Mutation. Evolution 53, 645 (1999).

27. Rowe, L. \& Houle, D. The lek paradox and the capture of genetic variance by condition dependent traits. Proc R Soc Lond B 263, 1415-1421 (1996).

28. Fox, C. W. Multiple Mating, Lifetime Fecundity and Female Mortality of the Bruchid Beetle, Callosobruchus maculatus (Coleoptera: Bruchidae). Funct. Ecol. 7, 203-208 (1993).

29. Fox, C. W., Stillwell, R. C., Wallin, W. G., Curtis, C. L. \& Reed, D. H. Inbreedingenvironment interactions for fitness: complex relationships between inbreeding depression and temperature stress in a seed-feeding beetle. Evol. Ecol. 25, 25-43 (2011).

30. Berger, D., Stångberg, J., Grieshop, K., Martinossi-Allibert, I. \& Arnqvist, G. Temperature effects on life-history trade-offs, germline maintenance and mutation rate under simulated climate warming. Proc R Soc B 284, 20171721 (2017).

31. Savalli, U. M. \& Fox, C. W. The effect of male size, age, and mating behavior on sexual selection in the seed beetle Callosobruchus maculatus. Ethol. Ecol. Evol. 11, 49-60 (1999).

32. Gay, L., Hosken, D. J., Vasudev, R., Tregenza, T. \& Eady, P. E. Sperm competition and maternal effects differentially influence testis and sperm size in Callosobruchus maculatus. J. Evol. Biol. 22, 1143-1150 (2009).

33. Eady, P. E. Why do male Callosobruchus maculatus beetles inseminate so many sperm? Behav. Ecol. Sociobiol. 36, 25-32 (1995).

34. Hotzy, C. \& Arnqvist, G. Sperm Competition Favors Harmful Males in Seed Beetles. Curr. Biol. 19, 404-407 (2009). 
521 35. Crudgington, H. S. \& Siva-Jothy, M. T. Genital damage, kicking and early death. Nature 407, 855-856 (2000).

36. Arnqvist, G., Nilsson, T. \& Katvala, M. Mating rate and fitness in female bean weevils. Behav. Ecol. 16, 123-127 (2005).

37. Fox, C. W. The influence of maternal age and mating frequency on egg size and offspring performance in Callosobruchus maculatus (Coleoptera: Bruchidae). Oecologia 96, 139_ (2007). maculatus (F.). Ecology 56, 696-702 (1975). 
44. Berger, D. et al. INTRALOCUS SEXUAL CONFLICT AND ENVIRONMENTAL STRESS: SEX, GENES, AND CONFLICT IN STRESSFUL ENVIRONMENTS. Evolution n/a-n/a (2014). doi:10.1111/evo.12439

45. Utida, S. Density dependent polymorphism in the adult of Callosobruchus maculatus (Coleoptera, Bruchidae). J. Stored Prod. Res. 8, 111-125 (1972).

46. Martinossi-Allibert, I., Thilliez, E., Arnqvist, G. \& Berger, D. Sexual selection, environmental robustness and evolutionary demography of maladapted populations: a test using experimental evolution in seed beetles. (2018). doi:10.1101/426056

47. Scharf, I. \& Martin, O. Y. Same-sex sexual behavior in insects and arachnids: prevalence, causes, and consequences. Behav. Ecol. Sociobiol. 67, 1719-1730 (2013).

48. Bates, D., Mächler, M., Bolker, B. \& Walker, S. Fitting Linear Mixed-Effects Models using lme4. ArXiv14065823 Stat (2014).

49. Sherry, D. F., Jacobs, L. F. \& Gaulin, S. J. Spatial memory and adaptive specialization of the hippocampus. Trends Neurosci. 15, 298-303 (1992).

50. Papaj, D. R. \& Prokopy, R. J. Ecological and Evolutionary Aspects of Learning in Phytophagous Insects. Annu. Rev. Entomol. 34, 315-350 (1989).

51. Corral-López, A. et al. Female brain size affects the assessment of male attractiveness during mate choice. Sci. Adv. 3, e1601990 (2017).

52. Kotrschal, A., Corral-Lopez, A., Amcoff, M. \& Kolm, N. A larger brain confers a benefit in a spatial mate search learning task in male guppies. Behav. Ecol. 26, 527-532 (2015).

53. Hollis, B. \& Kawecki, T. J. Male cognitive performance declines in the absence of sexual selection. Proc. R. Soc. B Biol. Sci. 281, 20132873-20132873 (2014).

54. Jacobs, L. F. Sexual selection and the brain. Trends Ecol. Evol. 11, 82-86 (1996).

55. Geary, D. C. Sexual selection and sex differences in spatial cognition. Learn. Individ. Differ. 7, 289-301 (1995). 
570 56. Lande, R. Sexual dimorphism, sexual selection, and adaptation in polygenic characters.

$571 \quad$ Evolution 34, 292-305 (1980).

572 57. Stewart, A. D. \& Rice, W. R. Arrest of sex-specific adaptation during the evolution of

573 sexual dimorphism in Drosophila. Nat. Ecol. Evol. 2, 1507-1513 (2018).

574 58. Chippindale, A. K., Gibson, J. R. \& Rice, W. R. Negative genetic correlation for adult

575 fitness between sexes reveals ontogenetic conflict in Drosophila. 5

576 59. Rowe, L., Chenoweth, S. F. \& Agrawal, A. F. The Genomics of Sexual Conflict. Am. Nat. 000-000 (2018). doi:10.1086/698198

60. Bonduriansky, R. \& Chenoweth, S. F. Intralocus sexual conflict. Trends Ecol. Evol. 24,

66. Snell-Rood, E. C., Davidowitz, G. \& Papaj, D. R. Reproductive tradeoffs of learning in a butterfly. Behav. Ecol. 22, 291-302 (2011). 280-288 (2009).

61. Berger, D., Berg, E. C., Widegren, W., Arnqvist, G. \& Maklakov, A. A. Multivariate intralocus sexual conflict in seed beetles: SEXUAL CONFLICT AND LIFE-HISTORY PLEIOTROPY. Evolution 68, 3457-3469 (2014).

62. Harano, T., Okada, K., Nakayama, S., Miyatake, T. \& Hosken, D. J. Intralocus sexual conflict unresolved by sex-limited trait expression. Curr. Biol. CB 20, 2036-2039 (2010).

63. Navarrete, A., van Schaik, C. P. \& Isler, K. Energetics and the evolution of human brain size. Nature 480, 91-93 (2011).

64. Mery, F. \& Kawecki, T. J. A Cost of Long-Term Memory in Drosophila. Science 308, $1148-1148$ (2005).

65. Snell-Rood, E. C., Papaj, D. R. \& Gronenberg, W. Brain Size: A Global or Induced Cost of Learning? Brain. Behav. Evol. 73, 111-128 (2009).

67. ller, A. P. M. \& Alatalo, R. V. Good-genes effects in sexual selection. Proc. R. Soc. B Biol. Sci. 266, 85 (1999). 
595 68. Kotrschal, A. et al. Positive genetic correlation between brain size and sexual traits in 596 male guppies artificially selected for brain size. J. Evol. Biol. 28, 841-850 (2015).

69. Buechel Séverine D., Booksmythe Isobel, Kotrschal Alexander, Jennions Michael D. \& Kolm Niclas. Artificial selection on male genitalia length alters female brain size. Proc. R. Soc. B Biol. Sci. 283, 20161796 (2016).

70. Emlen, D. J. Costs and the Diversification of Exaggerated Animal Structures. Science 291, 1534-1536 (2001).

71. Kodric-Brown, A. \& Brown, J. H. Truth in Advertising: The Kinds of Traits Favored by Sexual Selection. Am. Nat. 124, 309-323 (1984).

72. Grieshop, K. \& Arnqvist, G. Sex-specific dominance reversal of genetic variation for fitness. PLOS Biol. 16, e2006810 (2018).

73. Berger, D. et al. Intralocus Sexual Conflict and the Tragedy of the Commons in Seed Beetles. Am. Nat. 188, E98-E112 (2016). Ecol. Evol. 27, 511-519 (2012).

75. Lande, R. Models of speciation by sexual selection on polygenic traits. Proc. Natl. Acad. Sci. 78, 3721-3725 (1981). (2007).

78. Panhuis, T. M., Butlin, R., Zuk, M. \& Tregenza, T. Sexual selection and speciation. 58, 155-183 (1983). 
620 Figure 1: The experimental arena (a $150 \mathrm{~mm}$ diameter petri-dish) used for the behavioural 621 assays. The symbols indicate the following: white star = place where the four focal 622 individuals were placed at the start of each trail; red triangles $=$ the wrong choice (males for 623 male trails $\mid$ chick-peas for female trails), green circles $=$ the correct choice (females for male 624 trails | black-eyed beans for female trails).

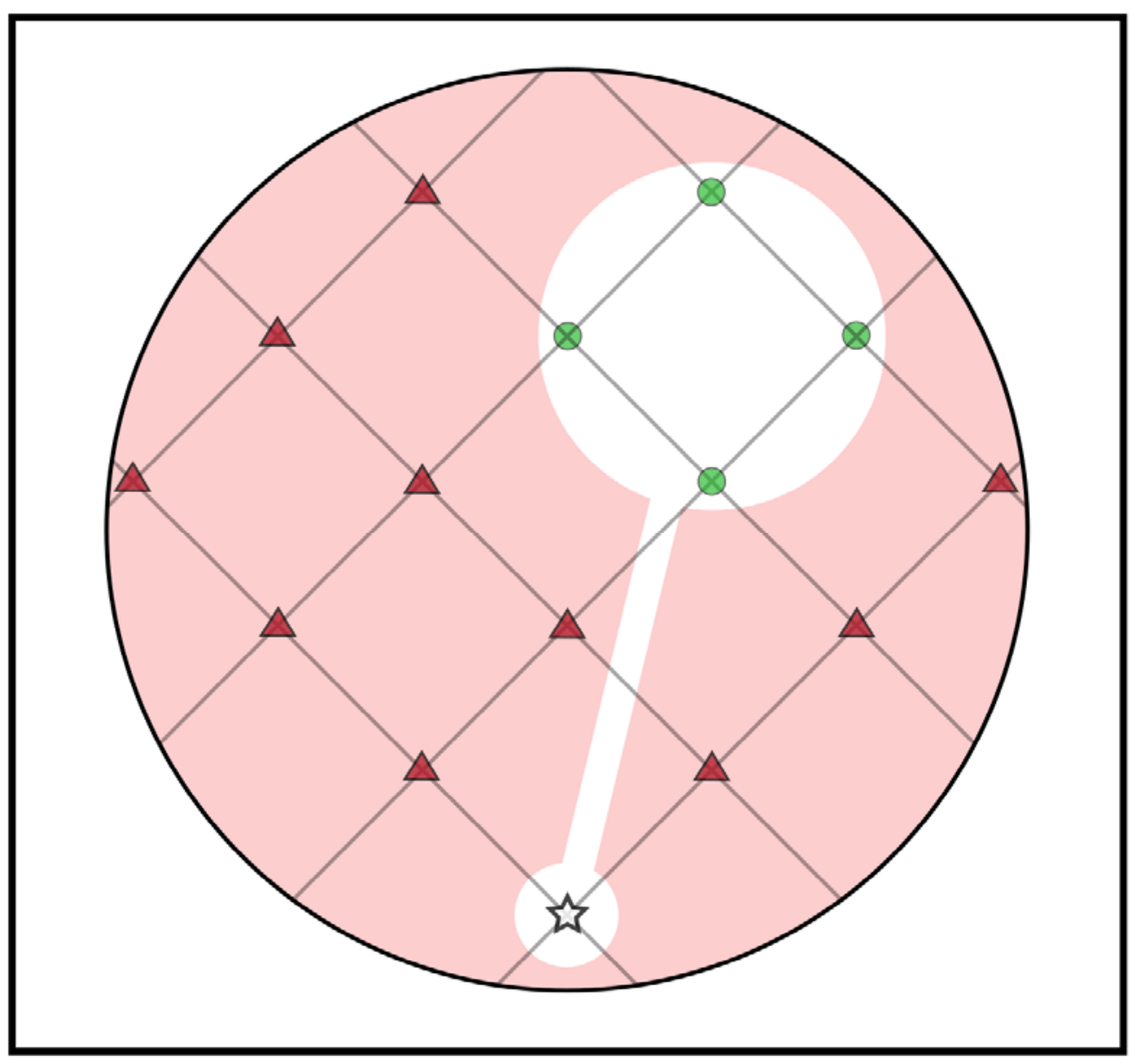


627 Figure 2: Sex-specific baseline cognitive performance and learning in the three replicate 628 monogamous (red) and polygamous (orange) lines. Male mate searching ability (A, C) and 629 female host searching ability (B, D) in terms of "error rates" (the fraction of male mating 630 attempts with other males, and the fraction of female contacts with the suboptimal host).

631 Shown are data for naïve beetles (A, B) in the first 10-minute trail, and trained beetles (C, D) 632 in the third 10-minute trail. The line type (thick and full to thin and hatched) designates the 633 line identity and makes it possible to match line performance across the first and the third 634 trail.

635

MALE MATE SEARCHING
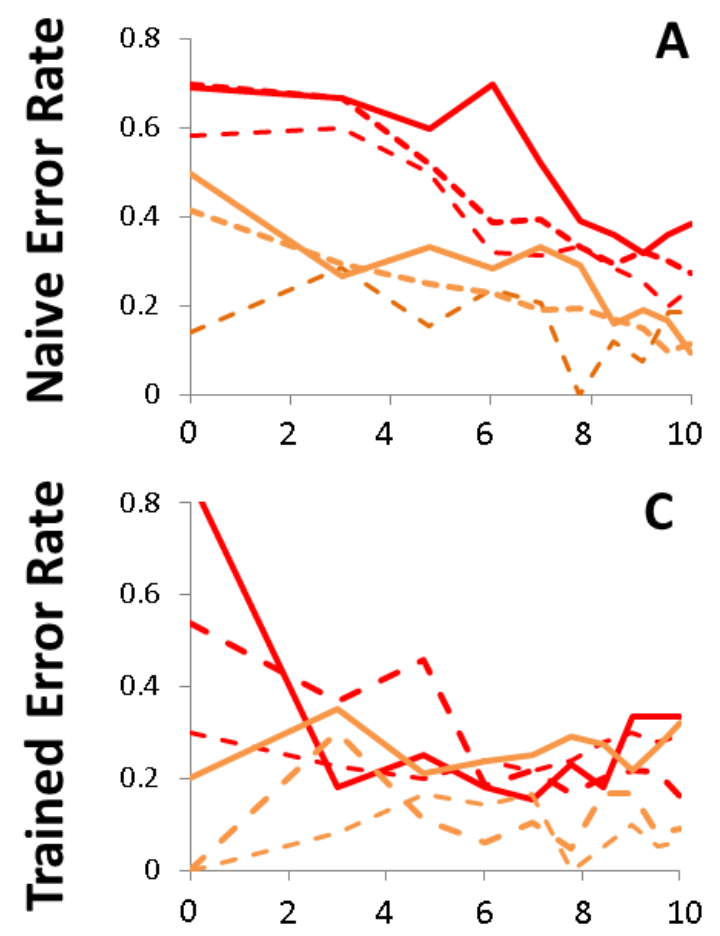

FEMALE HOST SEARCHING
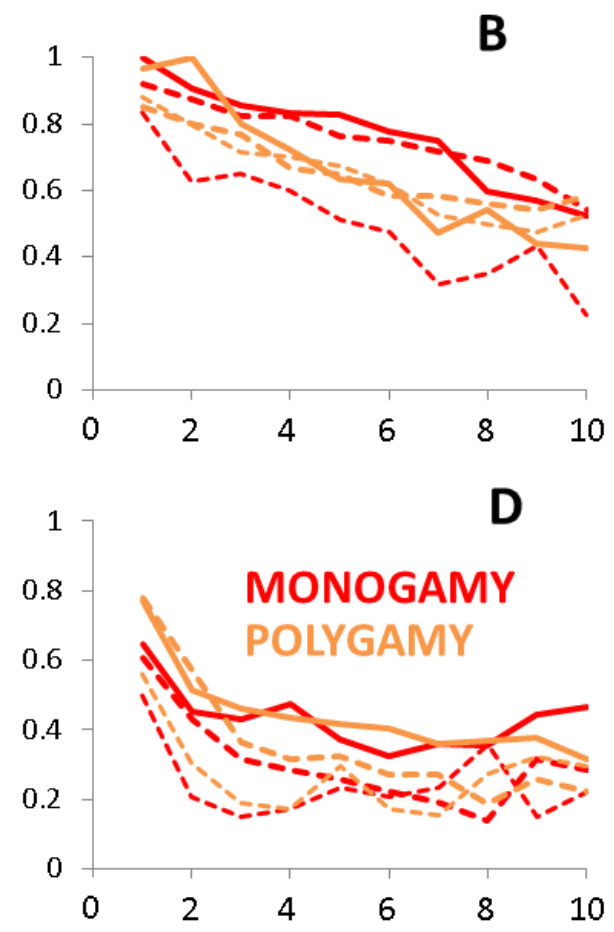
637 Figure 3: Cognitive performance (in terms of error rates) averaged over the full 10-minute 638 trial, for naïve beetles in the first trail (open symbols) and trained beetles in the third trail 639 (closed symbols). Mean male and female error rates are shown for each of the three replicate 640 monogamous (red) and polygamous (orange) lines.

641

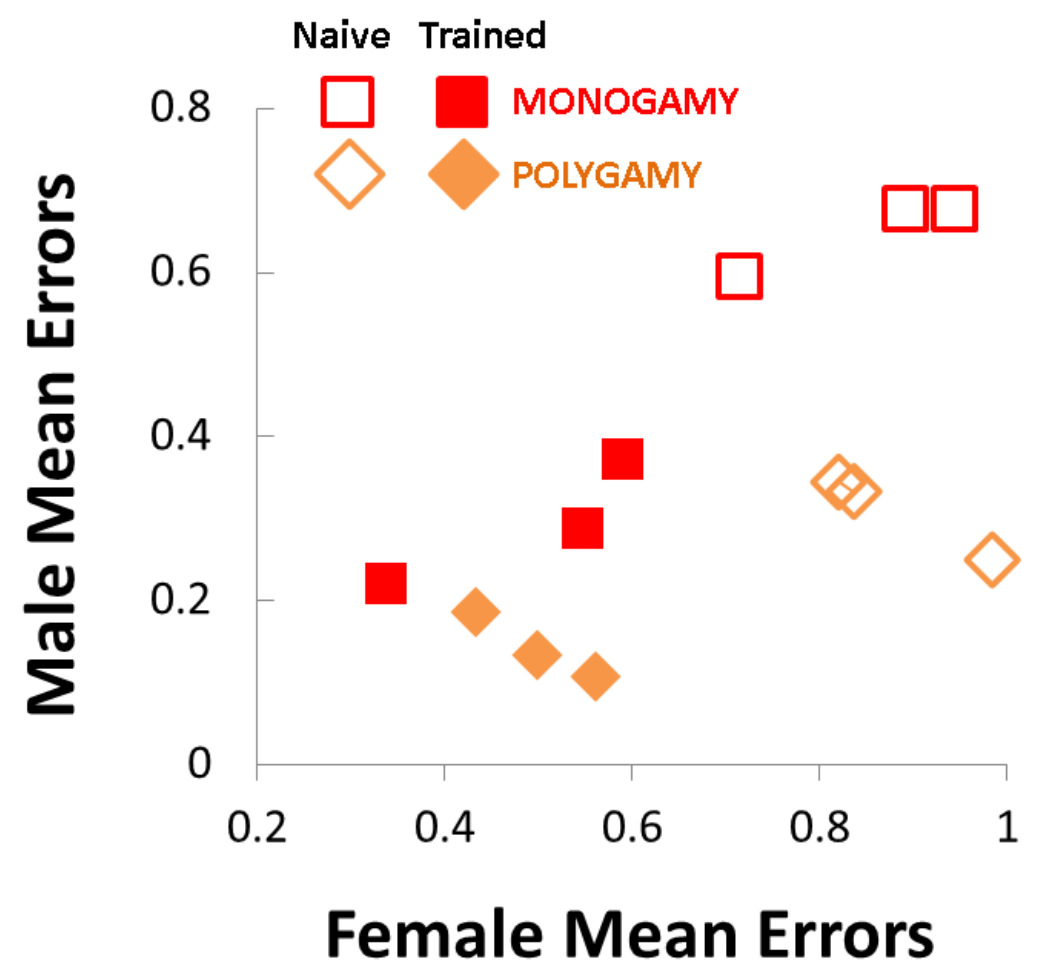


644 Figure 4: Sex-specific genetic correlations between cognitive performance and lifetime 645 reproductive success. Shown are correlations based on the 6 lines (3 from each regime) 646 between cognitive performance (error rates in the three consecutive behavioral trails and a 647 measure of learning from trail 1 to 3: [E1-E3]/E1), and lifetime reproductive success ( ) on 648 two host species (be $=$ ancestral black eyed beans, adz = adzuki beans). Within-sex genetic 649 correlations are highlighted by green $(\mathrm{F}=$ female $)$ and purple $(\mathrm{M}=$ male $)$ squares. Black 650 squares highlight between-sex genetic correlations. Full lines designate correlations between 651 measures of cognitive performance and hatched lines between cognitive performance and 652 lifetime reproductive success. Note that the same correlations are depicted both above and 653 below the diagonal. Circles on the diagonal are trait variances standardized to a size $=1$. 


\section{Figure S1: Experimental arenas}

661 Arenas used to assess cognition and learning. Shown are the two arena types with reversed 662 color schemes, placed on heating plates situated ca. 1 metre apart. Below the arenas are the $66330 \mathrm{~mm}$ diameter acclimation petri-dishes where the four focal beetles spent 20 minutes prior 664 to and in-between trails.
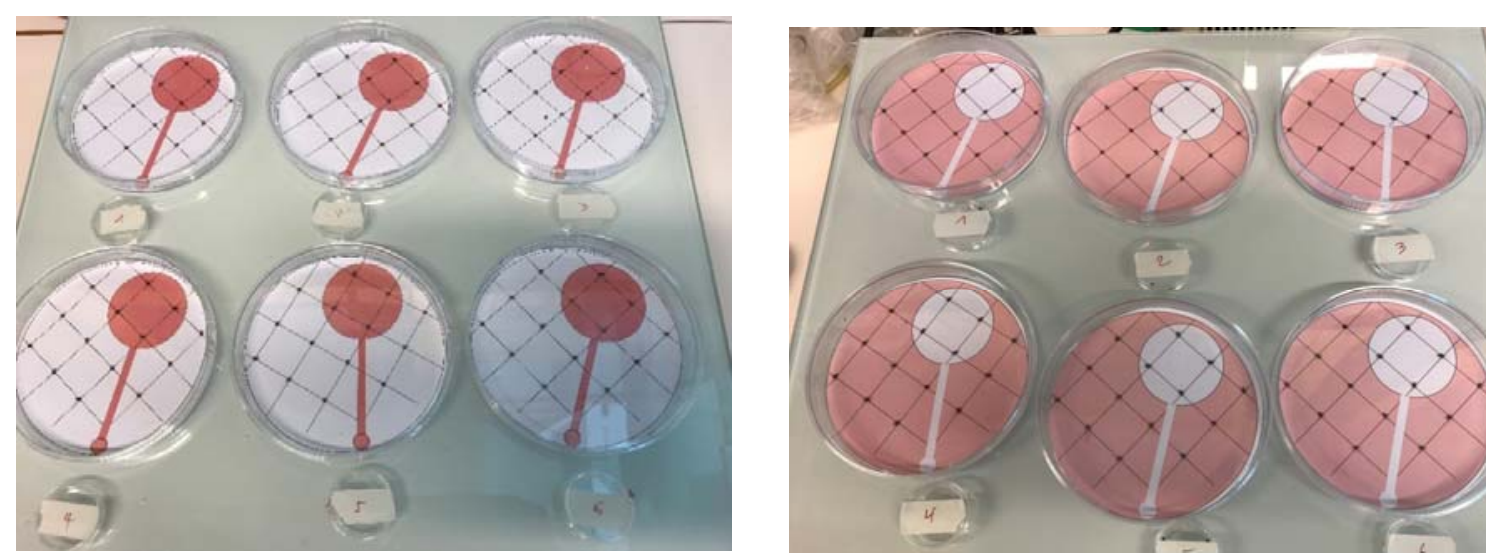
669 Figure S2: Male cognitive performance

670 The averaged summed number of times that the four males made mounting attempts on

671 females (left; A \& C) and males (right; B \& D) during the first ("Naïve" beetles) and third 672 ("Trained" beetles) behavioural trail.

673

FEMALES MOUNTED
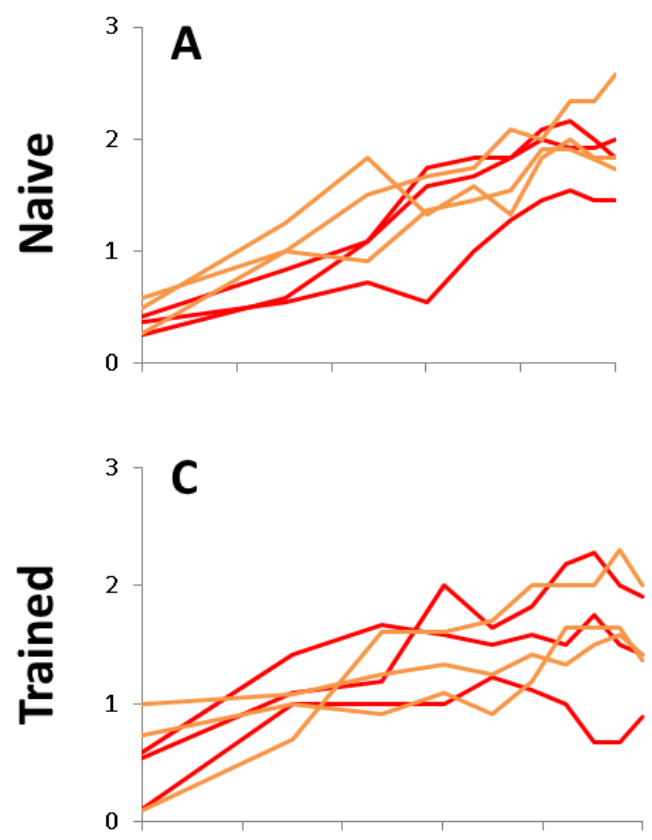

MALES MOUNTED
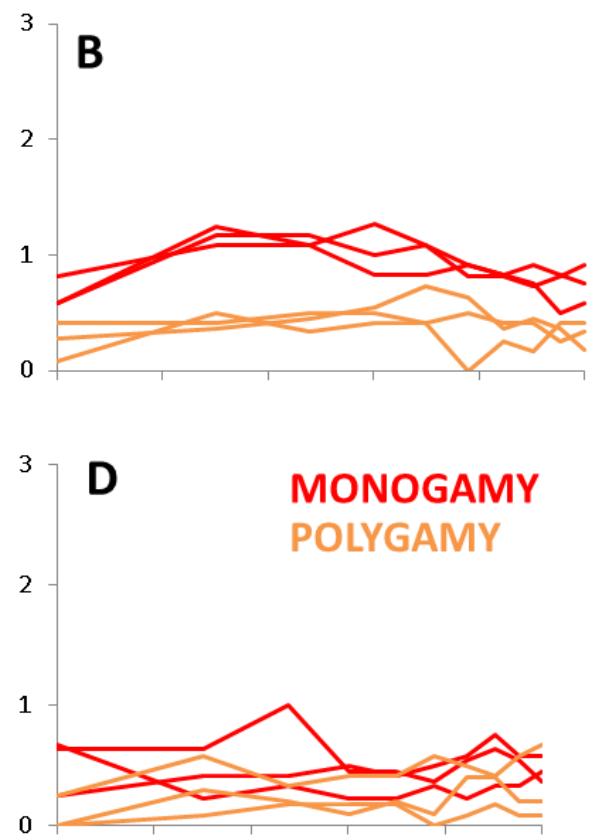

677 Table S2: Male cognitive performance

678 Type II Wald chi-square tests

\author{
trial \\ time \\ regime \\ trial:time \\ trial:regime \\ time:regime \\ trial: time:regime
}

\begin{tabular}{|c|c|c|c|}
\hline Chisq & $\mathrm{Df}$ & $\mathrm{P}$ & \\
\hline 4.2328 & 2 & 0.120466 & \\
\hline 80.0619 & 1 & $<2.2 e-16$ & $\star \star *$ \\
\hline 8.5645 & 1 & 0.003428 & * * \\
\hline 18.7180 & 2 & $8.619 e-05$ & $\star \star \star$ \\
\hline 3.8489 & 2 & 0.145959 & \\
\hline 2.3646 & 1 & 0.124115 & \\
\hline 0.1789 & 2 & 0.914416 & \\
\hline
\end{tabular}




\section{Figure S3: Female cognitive performance}

680 The averaged summed number of times that the four females made inspections of optimal 681 black-eyed beans (left; A \& C) and sub-optimal chick-peas (right; B \& D) during the first 682 ("Naïve" beetles) and third ("Trained" beetles).

683

OPTIMAL HOST VISITS
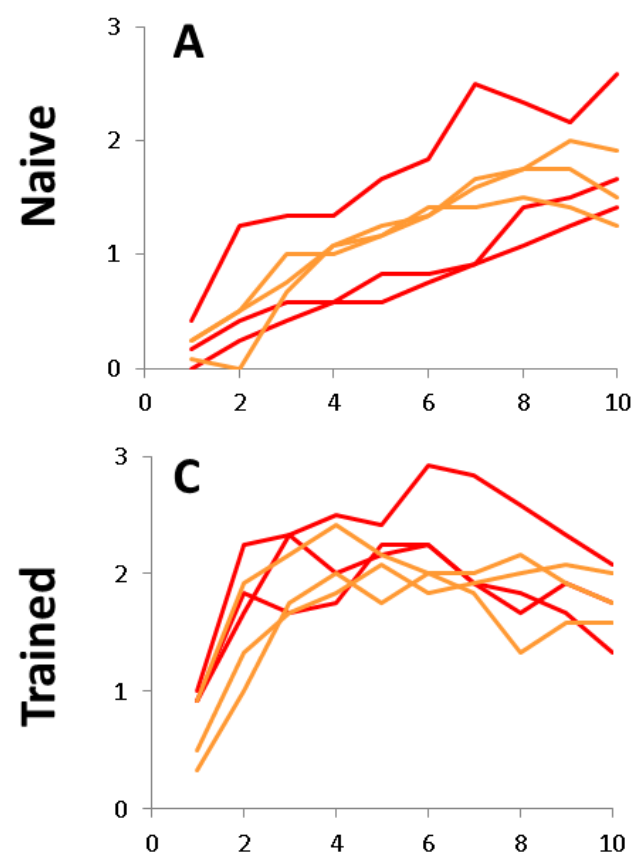

SUBOPTIMAL HOST VISITS
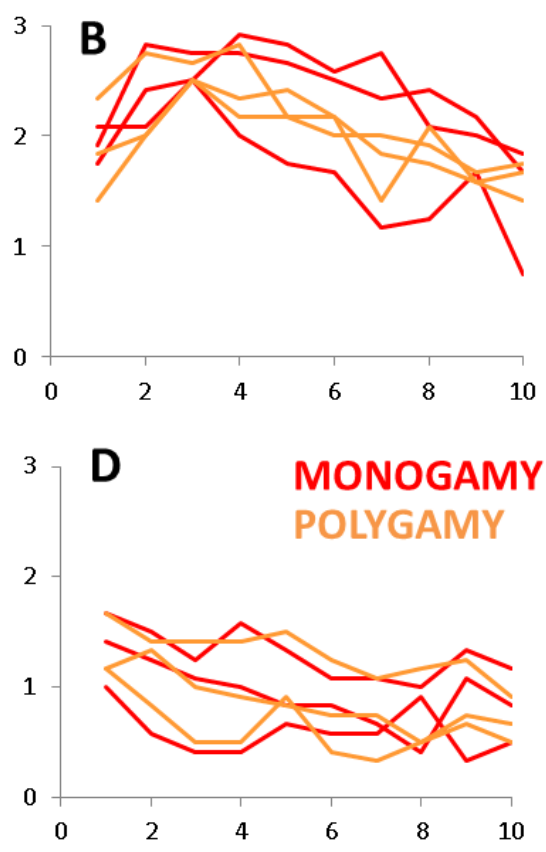

Table S3: Female cognitive performance

Type II Wald chi-square tests

trial

minuteLOG

sel

trial:minuteLOG

trial:sel

minuteLOG:sel

trial :minuteLOG:sel
Chisq Df

99.09672

$246.7218 \quad 1$

0.06141

33.76392

0.51652

$0.2564 \quad 1$

5.75612
$\mathrm{P}$

$<2.2 e-16 * \star *$

$<2.2 e-16 * * *$

0.80431

$4.659 e-08 * * *$

0.77240

0.61259

0.05624 
bioRxiv preprint doi: https://doi.org/10.1101/514711; this version posted January 9,2019 . The copyright holder for this preprint (which was not certified by peer review) is the author/funder, who has granted bioRxiv a license to display the preprint in perpetuity. It is made available under aCC-BY-NC-ND 4.0 International license.

\section{Table S4: Sex differences in cognitive performance}

689

\begin{tabular}{|c|c|}
\hline 690 & sex \\
\hline & trial \\
\hline 691 & $\begin{array}{l}\text { time } \\
\text { regime }\end{array}$ \\
\hline 692 & sex:trial \\
\hline 693 & $\begin{array}{l}\text { trial:time } \\
\text { sex:regime }\end{array}$ \\
\hline 694 & trial:regime \\
\hline 695 & $\begin{array}{l}\text { time:regime } \\
\text { sex:trial:time }\end{array}$ \\
\hline 696 & $\begin{array}{l}\text { sex:trial:sel } \\
\text { sex:time:regime }\end{array}$ \\
\hline 697 & $\begin{array}{l}\text { trial:time:regime } \\
\text { sex:trial:time:regime }\end{array}$ \\
\hline
\end{tabular}

698

699

$\begin{array}{rlcl}\text { Chisq } & \text { Df } & P & \\ 69.4112 & 1 & <2.2 e-16 & * * * \\ 64.0866 & 2 & 1.213 e-14 & * * * \\ 375.9454 & 1 & <2.2 e-16 & * * * \\ 1.1411 & 1 & 0.285412 & \\ 21.9326 & 2 & 1.727 e-05 & * * * \\ 12.3163 & 1 & 0.000449 & * * * \\ 52.6199 & 2 & 3.747 e-12 & * * * \\ 5.9118 & 1 & 0.015040 & \text { * } \\ 3.1970 & 2 & 0.202196 & \\ 0.1909 & 1 & 0.662209 & \\ 0.4299 & 2 & 0.806599 & \\ 2.0383 & 2 & 0.360893 & \\ 2.3276 & 1 & 0.127096 & \\ 3.6235 & 2 & 0.163366 & \\ 2.7250 & 2 & 0.256015 & \end{array}$

